\title{
Recovery of Swallowing
}

\author{
Nam-Jong Paik and Won-Seok Kim
}

\section{Introduction}

Dysphagia is one of the common impairments after stroke, which ranges widely from $29 \%$ to $81 \%$ according to the timing of the survey after stroke or the methods used for dysphagia assessment (e.g., questionnaire, clinical examination, instrumental test) (Martino et al. 2005). Pharyngeal phase dysphagia is more prevalent (Han et al. 2001) and brain stem or specific cortical lesions may attribute more for poststroke dysphagia (Galovic et al. 2013; Martino et al. 2005). Dysphagia is associated with the higher risk of non-silent or silent aspirations and aspiration pneumonia (Ding and Logemann 2000; Falsetti et al. 2009; Perry and Love 2001). In addition, dysphagia is associated with other medical complications such as dehydration, malnutrition, mortality, and recovery after stroke (Perlman 1996). Therefore, detecting and managing dysphagia after stroke could prevent medical complications and reduce the length of hospital stay and socioeconomic burdens (Smithard et al. 1996).

In this chapter, we reviewed the clinical evidence related with dysphagia assessment and management after stroke. The topics selected are: (1) Dysphagia screening; (2) Instrumental assessment of dysphagia; (3) Treatment of dysphagia including behavioral interventions, neuromuscular electrical stimulation, noninvasive brain stimulation, and oral hygiene; and (4) Enteral tube feedings. Five clinical practice guidelines were selected for the review and adaptation (Boddice et al. 2010; Hebert et al. 2016; Smith 2010; Winstein et al. 2016; Kim et al. 2016). Articles published frvom 2014 to 2017 were additionally searched in the PubMed and Cochrane Library using the MeSH keywords "(cerebrovascular disorders OR stroke OR cerebral hemorrhage OR cerebral infarction) AND (Dysphagia OR Deglutition OR oral hygiene)," and in the EMBASE using EMTREE with the keywords, "(cerebrovascular disease)

N.-J. Paik $(\bowtie)$ · W.-S. Kim

Department of Rehabilitation Medicine, Seoul National University College of Medicine,

Seoul National University Bundang Hospital, Seongnam, Republic of Korea

e-mail: njpaik@snu.ac.kr 
AND (Dysphagia OR swallowing disorder OR deglutition).” Total 2118 articles were found by these electronic searches. Titles and abstracts were reviewed for screening and non-English papers, commentaries, case series, narratives, book chapters, editorials, nonsystematic reviews, and conference papers were excluded. Two independent raters screened all 2118 articles and any disagreement was resolved by discussion. In each topic, the systematic review, meta-analyses, and randomized controlled trials (RCTs) were considered for inclusion first, but if no articles were available for the topic, observational studies were additionally selected.

Systematic reviews and meta-analyses $(n=11)$, RCTs $(n=5)$, and observational studies $(n=10)$ relevant to each selected topic in dysphagia (31 articles in total including five clinical practice guidelines) were selected for full-text review for summarizing the currently best available evidence, assessing its overall quality of evidence and for the provision of recommendations. A complete list of selected references is presented online at www.clinical-pathways.org. The recommendations given in this chapter follow the same rules as outlined in chapter "Clinical Pathways in Stroke Rehabilitation: Background, Scope, and Methods" (see Chap. 2).

\section{Dysphagia Screening Early after Stroke}

One systematic review included 14 studies to detect oropharyngeal dysphagia with bedside screening in patients with neurological disorders. The sensitivity and specificity to detect aspiration ranged from $45 \%$ to $100 \%$, and $29 \%$ to $86 \%$, respectively (Kertscher et al. 2014). In one systematic review of swallowing screens after acute stroke, four screening protocols that met the quality criteria showed high sensitivities $(\geq 87 \%)$ and specificities ( $\geq 91 \%$ ) (Schepp et al. 2011). A recent prospective, double-blind study in patients with stroke demonstrated that a dysphagia screen protocol has the high sensitivity $(96.5 \%)$ and acceptable specificity $(55.8 \%)$ as a screening tool to detect aspiration (Warnecke et al. 2017). Multiple observational multicenter prospective or registry-based studies with large sample size showed that the screening failure of dysphagia after stroke is associated with higher risk of pneumonia or mortality (Hinchey et al. 2005; Joundi et al. 2017a; b; Lakshminarayan et al. 2010). Bedside screening for dysphagia by the trained nurses after acute stroke demonstrated the significant reduction in the time to dysphagia screening and pneumonia rate compared to the screening by speech therapists in the pre- and post-intervention trial (Palli et al. 2017).

Recommendation: Dysphagia screening ought to be administered by the trained healthcare provider early after stroke onset before starting oral feeding to prevent pneumonia and other adverse events such as malnutrition, dehydration, and to reduce the mortality (CEBM classification: level 1, GRADE quality: moderate, recommendation grade: $\mathrm{A}+$ ).

One of the dysphagia screening tools that showed acceptable reliability, higher sensitivity, and negative predictive value, which consists of items such as alertness assessment, dry swallowing test, and direct swallowing test with water or semisolid and solid foods, should be used (CEBM classification: level 1, Grade quality: moderate, recommendation: $\mathrm{B}+$ ). 


\section{Instrumental Assessments to Detect Dysphagia or Aspiration}

A bedside screening test alone has a limitation to assess dysphagia after stroke because patients with stroke can aspirate without overt clinical signs or symptoms (silent aspiration), and the assessment for sequential swallowing function (oral, pharyngeal, and esophageal phase) is difficult by a bedside screening tool (Singh and Hamdy 2006). The widely used instrumental assessments for dysphagia are videofluoroscopic swallowing study (VFSS) or fiberoptic endoscopic evaluation of swallowing (FEES), which have advantages to assess the physiological or structural causes of dysphagia necessary for providing the appropriate instruction for dysphagia management (e.g., swallow therapy, compensation maneuver, dietary modification). In a systematic review including 24 observational studies, dysphagia incidence after stroke was higher with the VFSS (64-78\%) than those with bedside screening or structured clinical assessments (37-55\%) (Martino et al. 2005). High silent aspiration rate (67\% of silent aspiration among patients with aspiration) detected by VFSS in one observational study with acute stroke patients indicated that the clinical bedside test alone could not predict the risk of aspiration or pneumonia (Daniels et al. 1998). In a prospective observational study to compare the bedside screening test versus VFSS in patients with acute stroke, $14 \%$ of patients who passed the screening test showed aspiration in VFSS and diet recommendations were changed to the more conservative level in $28 \%$ of the patients after VFSS (Leigh et al. 2016). One retrospective observational study showed that selective FEES significantly reduced pneumonia (Bax et al. 2014). However, no high-quality study has shown that the instrumental test can reduce the pneumonia rate significantly more than the bedside screening test (Doggett et al. 2001; Kjaersgaard et al. 2014), although one recent study using a decision-analysis model from multiple data sources including meta-analyses and other relevant clinical studies demonstrated that VFSS screening for dysphagia was more cost-effective than a bedside examination alone or a combination of bedside examination with VFSS (Wilson and Howe 2012). The superiority of one method to another between VFSS and FEES was not significant and both can be applied according to the clinical situations (Aviv 2000; Boddice et al. 2010; Hebert et al. 2016; Smith 2010).

Recommendation: Instrumental assessments such as VFSS or FEES ought to be performed to verify the aspiration and to set the appropriate dysphagia management plan in stroke patients who showed the risk for pharyngeal dysphagia or aspiration in the initial or ongoing swallowing screens (CEBM classification: level 1, GRADE quality: moderate, recommendation grade: $\mathrm{A}+$ ).

\section{$4 \quad$ Treatment of Dysphagia}

Early and comprehensive treatment of dysphagia is important, if the dysphagia is detected by the screening or instrumental tests. Conventional dysphagia treatment usually includes the swallowing exercise, compensatory techniques, and 
appropriate dietary modifications. In this chapter, the evidence for behavioral intervention programs including the conventional dysphagia treatment components were reviewed, because these components are usually provided by rather a program than an isolated component, and the effects of each isolated component cannot be evaluated sufficiently. Based on the current evidence, the evidence for neuromuscular electrical stimulation (NMES), which is commonly used with the behavioral interventions, was reviewed. And since meta-analyses of acupuncture studies were available, the reviews of evidence and recommendation for acupuncture were included. Noninvasive brain stimulations such as repetitive transcranial magnetic stimulation (rTMS) or transcranial direct current stimulation (tDCS) for dysphagia were also reviewed for their evidence because of presence of more recent RCTs and systematic reviews. Lastly, the evidence of oral hygiene to prevent pneumonia in stroke patients with dysphagia was reviewed.

\subsection{Behavioral Interventions}

One RCT investigated the effect of behavioral interventions on dysphagia in acute stroke with the primary outcome of survival free of an abnormal diet at 6 months (Carnaby et al. 2006). Patients were randomly allocated to usual care $(n=102)$, standard low-intensity intervention $(n=102)$ consisting of compensation strategies (e.g., environmental modification such as the upright positioning for feeding), safe swallowing advice, and appropriate dietary modification, three times per week for a month or standard high-intensity intervention $(n=102)$ comprising direct swallowing exercises and appropriate dietary modification, every working day for a month. A significant reduction in swallowing-related medical complications $(\mathrm{RR}=0.73$, 95\% CI: 0.6 to 0.9 ), chest infection ( $\mathrm{RR}=0.56,95 \% \mathrm{CI}$ : 0.4 to 0.8 ), death or institutionalization ( $R R=0.73,95 \%$ CI: 0.55 to 0.97$)$, and a significant increased proportion of patients who recovered from swallowing ( $R R=1.41,95 \% \mathrm{CI}: 1.03$ to 1.94) were verified in the standard intervention compared to the usual care. Further, a dose-response relation was also apparent with improved recovery of swallowing function and less chest infection in patients receiving intensive standard swallowing therapy compared to less intensive standard therapy and usual care.

One systematic review including 15 RCTs with a broad range of swallowing treatment demonstrated that the general dysphagia treatment programs were related with a less risk of pneumonia in patients with acute stroke (Foley et al. 2008). In a Cochrane systematic review including five studies with behavioral interventions $(n=423)$ consisting of swallowing exercise, environment modifications, and appropriate dietary modification, behavioral interventions reduced the dysphagia at the end of trial $(t=5 ; n=423 ; \mathrm{OR}=0.52 ; 95 \% \mathrm{CI}$ : 0.30 to 0.88$)$; in addition, a nonsignificant reduction in chest infection/pneumonia was noted $(t=5 ; n=423$; OR 0.50; 95\% CI 0.24 to $1.04 ; \mathrm{I} 2=34 \% ; P=0.06)$, but not for case fatality $(t=2 ; n=306$; OR $0.83 ; 95 \%$ CI 0.46 to 1.51 ) or institutionalization ( $t=2 ; n=306$; OR $0.76 ; 95 \%$ CI 0.39 to 1.48) (Geeganage et al. 2012). Risk of bias assessment indicated a considerable proportion of unclear or high risk of bias of included studies. 
Recommendation: Dysphagia treatment program including behavioral interventions should be provided for the dysphagia patients after stroke to prevent dysphagiarelated complications and to recover the swallowing (CEBM classification: level 1, GRADE quality: moderate, recommendation grade: $\mathrm{B}+$ ).

\subsection{Neuromuscular Electrical Stimulation (NMES)}

One systematic review investigated the effect of NMES on the post-stroke dysphagia (Chen et al. 2016). Six RCTs involving 243 patients with stroke were included for meta-analysis to compare the effect of swallowing treatment with NMES and swallowing treatment without NMES. Swallowing treatment with NMES showed better recovery immediately after the intervention than the swallowing treatment without NMES, with a significant standardized difference of 1.27 (95\% CI: 0.51 to 2.02). However, the outcome measures used and the results in included studies were heterogeneous $\left(I^{2}=85 \%\right)$ and long-term effect could not be analyzed. A subgroup analysis suggested that both patients with acute/subacute and with chronic stroke might benefit. Treatment regimens varied with 20- to 60-min sessions applied 3 to 5 days per week for 2 to 4 weeks. A recent randomized controlled trial in subacute stroke patients $(n=162)$ with dysphagia (penetration aspiration score (PAS) in VFSS $\geq 3$ ) demonstrated no significant effect of NMES for three consecutive days compared to the sham NMES (Bath et al. 2016). The mean treatment current of NMES was $14.8 \pm 7.9 \mathrm{~mA}$ and duration was $9.9 \pm 1.2 \mathrm{~min}$ per session, which may be a suboptimal stimulation level. In another recent RCT, 82 patients with dysphagia after acute medullary infarction were allocated to three intervention groups: traditional swallowing treatment, swallowing treatment with NMES of sensory approach, and swallowing treatment with NMES of motor approach. NMES were for $20 \mathrm{~min}$, twice a day, for 5 days/week, over a 4-week period (Zhang et al. 2016). Both sensory and motor NMES with traditional swallowing treatment showed significantly greater improvement than the traditional swallowing treatment alone, and sensory NMES showed better improvement than the motor NMES.

Recommendation: NMES can be used to improve the swallowing function in patients with dysphagia after stroke, combined with a traditional swallowing treatment program including behavioral interventions (CEBM classification: level 1, GRADE quality: low, recommendation grade: 0).

\subsection{Acupuncture}

Acupuncture has been used as a complementary intervention for stroke recovery in some countries, although its mechanism of action is unclear (Zhang et al. 2014). In a recent Cochrane review, four acupuncture RCTs involving 256 patients were included for the meta-analysis with the outcome of dysphagia at the end of trial, which showed the significant favorable effect $(\mathrm{OR}=0.24,95 \% \mathrm{CI}$ : 0.13 to 0.46$)$ (Geeganage et al. 2012). In a recent systematic review, efficacy of acupuncture was analyzed with the 
pooling data from 71 RCTs involving 6010 patients, which showed the superior effect over the control ( $\mathrm{RR}=1.17,95 \% \mathrm{CI}$ : 1.13 to 1.21$)$ (Ye et al. 2017). Although, the pooled effect from these meta-analyses showed the positive effect of acupuncture for the swallowing recovery after stroke, most of the included RCTs in the systematic review by Ye et al. were conducted with small sample sizes and risk of bias (i.e., detection, performance, attrition and reporting bias) was high or unclear for most of the studies included (Ye et al. 2017). In addition, the included RCTs may have been confounded due to the use of routine acupuncture or a different type of acupuncture as control and the variations in the delivery of therapy (Geeganage et al. 2012).

Recommendation: Acupuncture can be considered to treat post-stroke dysphagia. (CEBM classification: level 1, GRADE quality: low, recommendation grade: 0 ).

\subsection{Noninvasive Brain Stimulation (NIBS)}

Recovery of swallowing after stroke is associated with the reorganization of the swallowing motor cortex (Hamdy et al. 2000). NIBS, such as repetitive transcranial magnetic stimulation (rTMS) or transcranial direct current stimulation (tDCS), is expected to modulate the swallowing motor cortex reorganization. Traditionally, the concept of interhemispheric rivalry is applied to decide the stimulation site or parameters for NIBS after stroke (Hummel and Cohen 2006) but stimulating either hemisphere can theoretically improve the swallowing because the swallowing musculature is dually innervated by both hemispheres (Hamdy et al. 1996). In recent years, the number of clinical trials investigating the effect of NIBS on post-stroke dysphagia has increased. One recent meta-analysis and systematic review including six RCTs (three rTMS and three tDCS studies) demonstrated that NIBS showed significant improvement of dysphagia compared with the sham stimulation (standardized mean difference $=1.08,95 \%$ CI: 0.29 to 1.88 ) (Yang et al. 2015). In a subgroup analysis, only rTMS showed significant beneficial effect and no statistically significant difference was found between the stimulation sites (ipsilesional vs. contralesional). No complications of NIBS were reported in these studies. Another systematic review included seven RCTs (four rTMS and three tDCS studies) (Pisegna et al. 2016). A pooled analysis of eight studies showed a significant, moderate pooled effect size $(0.55,95 \%$ CI: 0.17 to 0.93$)$. Studies stimulating the contralesional hemisphere showed numerically a somewhat bigger effect size $(0.65,95 \%$ CI: 0.14 to 1.16$)$ than that of studies stimulating ipsilesional hemisphere $(0.46,95 \%$ CI: 0.17 to 0.93 ). The long-term effect with pooled analysis of three studies was not statistically significant. A very recent systematic review including only six RCTs with a total of 163 acute and subacute stroke patients with dysphagia investigating rTMS effect showed a significant effect size of 1.24 (95\% CI: 0.67 to 1.81) for dysphagia outcome and effect was maintained until 4 weeks after the last session of rTMS (Liao et al. 2017). In a subgroup analysis, both high $(\mathrm{SMD}=1.38,95 \%$ $\mathrm{CI}=0.47$ to 2.29$)$ and low frequency stimulation $(\mathrm{SMD}=1.02,95 \% \mathrm{CI}=0.51$ to 1.53 ) were significantly effective. Stimulating the contralesional $(\mathrm{SMD}=0.91,95 \%$ $\mathrm{CI}=0.48$ to 1.35$)$ or bilateral hemisphere $(\mathrm{SMD}=1.60,95 \% \mathrm{CI}=0.57$ to 2.63$)$ was 
effective but ipsilesional stimulation yielded more variable effects and therefore was not significantly effective $(\mathrm{SMD}=1.59,95 \% \mathrm{CI}=-0.14$ to 3.31 ) in a subgroup analysis. Based on these recent systematic reviews and meta-analyses, NIBS in the form of rTMS may be effective on post-stroke dysphagia early after stroke (within 3 months), but the included studies were heterogeneous in terms of stimulation site, mode of stimulation and outcomes, which requires further well-designed clinical trials with larger sample sizes. As a note of caution, international safety standards (Rossi et al. 2009), individual exclusion criteria for rTMS, and medico-legal aspects related to the rTMS device used all need to be taken into consideration.

Recommendation: rTMS can be considered to treat post-stroke dysphagia by the clinical expert in this field as an additional treatment modality to the traditional swallowing treatment, especially within 3 months post-stroke (CEBM classification: level 1, GRADE quality: low, recommendation grade: 0 ).

\subsection{Oral Hygiene}

Bacterial species colonized in the oral cavity has been suggested as one of the important sources of pathogens for respiratory infections (Scannapieco et al. 2003). Patients with stroke may have poor oral hygiene due to the limitation of basic activities of daily living. One systematic review included four RCTs to investigate the effect of oral hygiene on pneumonia or respiratory tract infection in elderly people living in the institutions and reported positive preventive effects of oral hygiene on pneumonia and respiratory tract infection with absolute risk reductions from $6.6 \%$ to $11.7 \%$ (Sjögren et al. 2008). A prospective-controlled trial allocated patients with acute stroke to three groups: intervention (dysphagia screening with Gugging Swallowing Screen and intensified oral hygiene, $n=58)$, internal $(n=58)$, and external control groups (clinical dysphagia screening plus usual care) $(n=30)$ (Sørensen et al. 2013). The incidence of X-ray verified pneumonia was significantly lower in the intervention group (7\%), compared with internal $(28 \%)$ or external control groups (27\%). A recent pre- to post-intervention (oral hygiene care) trial with the historical control ( $n=707$ in the historical control, $n=949$ in the intervention group) in hospitalized patients with acute stroke demonstrated that the oral hygiene care significantly reduced the hospital-acquired pneumonia after adjusting for possible confounders ( $\mathrm{OR}=0.71,95 \% \mathrm{CI}$ : 0.51 to 0.98 ) (Wagner et al. 2016).

Recommendations: Oral hygiene care should be implemented to reduce the risk of pneumonia after stroke (CEBM classification: level 2, GRADE quality: moderate, recommendation grade: $\mathrm{B}+)$.

\section{$5 \quad$ Enteral Tube Feedings}

In stroke patients with dysphagia who cannot eat orally safely, enteral tube feeding is often required to provide enough nutrition for short or prolonged periods after stroke. Therefore, the decision on timing and maintenance of enteral feeding 
and type of enteral feeding (e.g., nasogastric tube or percutaneous gastrostomy) is important. In the well-designed, large sample-sized pragmatic multicenter randomized controlled trial, Feed or Ordinary Diet (FOOD) trials, the effect of timing and method of enteral tube feeding for stroke patients with dysphagia was investigated (Dennis et al. 2005). Dysphagic patients early after stroke who were uncertain when to start enteral tube feeding by the clinician were randomly assigned to early enteral tube feeding $(n=429)$ or avoidance of enteral tube feeding for more than 7 days $(n=428)$. Although it was not statistically significant, early enteral tube feeding showed the trend of reduction in risk of death of $5.8 \%$ (95\% CI: -0.8 to 12.5 ), but not for death or poor outcome (modified Rankin scale 4 or 5 ) of $1.2 \%$ ( -4.2 to 6.6 ). In the same trial, 321 dysphagic patients who require enteral feeding by the clinician's decision were randomly allocated to early percutaneous gastrostomy $(n=162)$ or nasogastric tube $(n=159)$. Early percutaneous gastrostomy showed the trend of increase in death or poor outcome of $7.8 \%$ (95\% CI: 0.0 to 15.5 ), but not for risk of death alone, i.e., $1.0 \%, 95 \% \mathrm{CI}:-10.0$ to 11.9. Therefore, early gastrostomy cannot be advocated based on this study. In a recent Cochrane systematic review including a small number of studies with small number of total subjects for the pooled analysis, gastrostomy showed better results compared with nasogastric tube feeding, in terms of treatment failure, feed delivery, and albumin concentration (Geeganage et al. 2012). Therefore, if oral intake is not possible after stroke for a prolonged period, it is reasonable to change the nasogastric tube feeding to tube feeding via gastrostomy. Individual factors are also taken into consideration, e.g., a nasogastric tube vs. percutaneous can create a different degree of discomfort for individuals and their family, and some individuals are more prone to risk for accidental nasogastric tube displacement and hence aspiration.

Recommendation: Early enteral tube feeding should be implemented in stroke patients with dysphagia who cannot swallow safely and intake sufficient nutrition orally. (CEBM classification: level 1, GRADE quality: moderate, recommendation grade: $\mathrm{B}+$ ). Early gastrostomy within 4 weeks after stroke does not have to be prioritized over nasogastric tube feeding, unless there is a mandatory reason for percutaneous gastrostomy (CEBM classification: level 1, GRADE quality: low, recommendation grade: 0 ). Percutaneous gastrostomy placement should be considered in stroke patients with dysphagia who require enteral tube feeding for a prolonged period (more than 4 weeks) (CEBM classification: level 1, GRADE quality: moderate, recommendation grade: $\mathrm{B}+$ ).

\section{Summary}

A decision tree for the rehabilitation of swallowing after acute stroke is suggested in Fig. 1. 


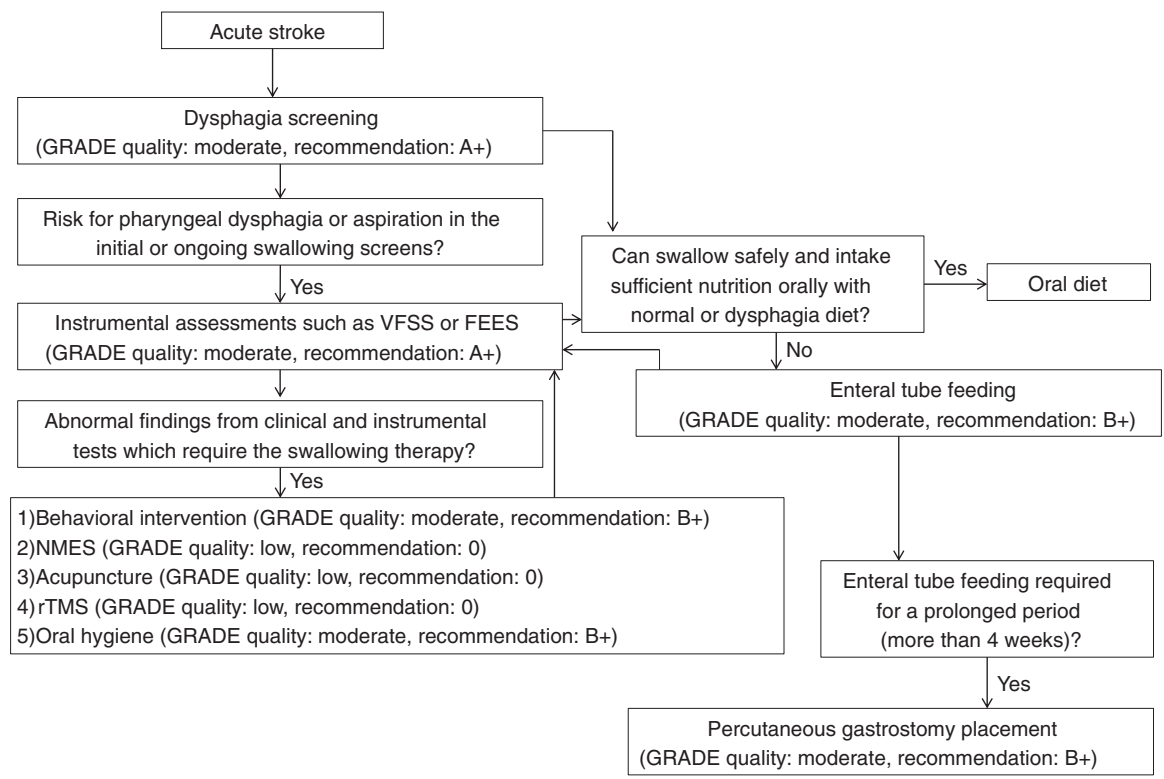

Fig. 1 A decision tree for the rehabilitation of swallowing after acute stroke. VFSS videofluoroscopic swallowing study, FEES fiberoptic endoscopic evaluation of swallowing, NMES neuromuscular electrical stimulation, $r T M S$ repetitive transcranial magnetic stimulation

\section{References}

Aviv JE (2000) Prospective, randomized outcome study of endoscopy versus modified barium swallow in patients with dysphagia. Laryngoscope 110:563-574

Bath PM, Scutt P, Love J et al (2016) Pharyngeal electrical stimulation for treatment of dysphagia in subacute stroke. Stroke 47(6): 1562-1570

Bax L, McFarlane M, Green E, Miles A (2014) Speech-language pathologist-led fiberoptic endoscopic evaluation of swallowing: functional outcomes for patients after stroke. J Stroke Cerebrovasc Dis 23(3):e195-e200

Boddice G, Brauer S, Gustafsson L, Kenardy J, Hoffmann T (2010) National Stroke Foundation: Clinical Guidelines for Stroke Management 2010. Melbourne, Australia: National Stroke Foundation, ISSBN0-978-0-9805933-3-4

Carnaby G, Hankey GJ, Pizzi J (2006) Behavioural intervention for dysphagia in acute stroke: a randomised controlled trial. Lancet Neurol 5(1):31-37

Chen YW, Chang KH, Chen HC, Liang WM, Wang YH, Lin YN (2016) The effects of surface neuromuscular electrical stimulation on post-stroke dysphagia: a systemic review and metaanalysis. Clin Rehabil 30(1):24-35

Daniels SK, Brailey K, Priestly DH, Herrington LR, Weisberg LA, Foundas AL (1998) Aspiration in patients with acute stroke. Arch Phys Med Rehabil 79:14-19

Dennis MS, Lewis SC, Warlow C, FOOD Trial Collaboration (2005) Effect of timing and method of enteral tube feeding for dysphagic stroke patients (FOOD): a multicentre randomised controlled trial. Lancet 365:764-772

Ding R, Logemann JA (2000) Pneumonia in stroke patients: a retrospective study. Dysphagia 15:51-57 
Doggett DL, Tappe KA, Mitchell MD, Chapell R, Coates V, Turkelson CM (2001) Prevention of pneumonia in elderly stroke patients by systematic diagnosis and treatment of dysphagia: an evidence-based comprehensive analysis of the literature. Dysphagia 16:279-295

Falsetti P, Acciai C, Palilla R et al (2009) Oropharyngeal dysphagia after stroke: incidence, diagnosis, and clinical predictors in patients admitted to a neurorehabilitation unit. J Stroke Cerebrovasc Dis 18:329-335

Foley N, Teasell R, Salter K, Kruger E, Martino R (2008) Dysphagia treatment post stroke: a systematic review of randomised controlled trials. Age Ageing 37:258-264

Galovic M, Leisi N, Müller M, Weber J, Abela E, Kägi G, Weder B (2013) Lesion location predicts transient and extended risk of aspiration after supratentorial ischemic stroke. Stroke 44(10):2760-2767

Geeganage C, Beavan J, Ellender S, Bath PM (2012) Interventions for dysphagia and nutritional support in acute and subacute stroke. Cochrane Database Syst Rev 10:CD000323

Hamdy S, Aziz Q, Rothwell JC et al (1996) The cortical topography of human swallowing musculature in health and disease. Nat Med 2:1217-1224

Hamdy S, Rothwell JC, Aziz Q, Thompson DG (2000) Organization and reorganization of human swallowing motor cortex: implications for recovery after stroke. Clin Sci (Lond) 99:151-157

Han TR, Paik N-J, Park JW (2001) Quantifying swallowing function after stroke: a functional dysphagia scale based on videofluoroscopic studies. Arch Phys Med Rehabil 82:677-682

Hebert D, Lindsay MP, McIntyre A et al (2016) Canadian stroke best practice recommendations: stroke rehabilitation practice guidelines, update 2015. Int J Stroke 11:459-484

Hinchey JA, Shephard T, Furie K, Smith D, Wang D, Tonn S (2005) Formal dysphagia screening protocols prevent pneumonia. Stroke 36:1972-1976

Hummel FC, Cohen LG (2006) Non-invasive brain stimulation: a new strategy to improve neurorehabilitation after stroke? Lancet Neurol 5:708-712

Joundi RA, Martino R, Saposnik G, Giannakeas V, Fang J, Kapral MK (2017a) Dysphagia screening after intracerebral hemorrhage. Int J Stroke 1:1747493017729265

Joundi RA, Martino R, Saposnik G, Giannakeas V, Fang J, Kapral MK (2017b) Predictors and outcomes of dysphagia screening after acute ischemic stroke. Stroke 48:900-906

Kertscher B, Speyer R, Palmieri M, Plant C (2014) Bedside screening to detect oropharyngeal dysphagia in patients with neurological disorders: an updated systematic review. Dysphagia 29:204-212

Kim DY, Kim YH, Lee J et al (2016) Clinical practice guideline for stroke rehabilitation in Korea 2016. Brain Neurorehabil 10(Suppl 1)

Kjaersgaard A, Nielsen LH, Sjölund BH (2014) Randomized trial of two swallowing assessment approaches in patients with acquired brain injury: facial-oral tract therapy versus fibreoptic endoscopic evaluation of swallowing. Clin Rehabil 28:243-253

Lakshminarayan K, Tsai AW, Tong X et al (2010) Utility of dysphagia screening results in predicting poststroke pneumonia. Stroke 41:2849-2854

Leigh JH, Lim JY, Han M-K, Bae HJ, Kim WS, Paik NJ (2016) A prospective comparison between bedside swallowing screening test and videofluoroscopic swallowing study in post-stroke dysphagia. Brain Neurorehabil 9:e7

Liao X, Xing G, Guo Z et al (2017) Repetitive transcranial magnetic stimulation as an alternative therapy for dysphagia after stroke: a systematic review and meta-analysis. Clin Rehabil 31:289-298

Martino R, Foley N, Bhogal S, Diamant N, Speechley M, Teasell R (2005) Dysphagia after stroke: incidence, diagnosis, and pulmonary complications. Stroke 36:2756-2763

Palli C, Fandler S, Doppelhofer K et al (2017) Early dysphagia screening by trained nurses reduces pneumonia rate in stroke patients: a clinical intervention study. Stroke 48:2583-2585

Perlman AL (1996) Dysphagia in stroke patients. Semin Neurol 16:341-348

Perry L, Love CP (2001) Screening for dysphagia and aspiration in acute stroke: a systematic review. Dysphagia 16:7-18

Pisegna JM, Kaneoka A, Pearson WG, Kumar S, Langmore SE (2016) Effects of non-invasive brain stimulation on post-stroke dysphagia: a systematic review and meta-analysis of randomized controlled trials. Clin Neurophysiol 127:956-968 
Rossi S, Hallett M, Rossini PM, Pascual-Leone A, Safety of TMS Consensus Group (2009) Safety, ethical considerations, and application guidelines for the use of transcranial magnetic stimulation in clinical practice and research. Clin Neurophysiol 120:2008-2039

Scannapieco FA, Bush RB, Paju S (2003) Associations between periodontal disease and risk for nosocomial bacterial pneumonia and chronic obstructive pulmonary disease. A systematic review. Ann Periodontol 8:54-69

Schepp SK, Tirschwell DL, Miller RM, Longstreth W (2011) Swallowing screens after acute stroke. Stroke 43(3):869-871

Singh S, Hamdy S (2006) Dysphagia in stroke patients. Postgrad Med J 82:383-391

Sjögren P, Nilsson E, Forsell M, Johansson O, Hoogstraate J (2008) A systematic review of the preventive effect of oral hygiene on pneumonia and respiratory tract infection in elderly people in hospitals and nursing homes: effect estimates and methodological quality of randomized controlled trials. J Am Geriatr Soc 56:2124-2130

Smith L (2010) Management of Patients with Stroke: rehabilitation, prevention and Management of Complications, and discharge planning: a National Clinical Guideline. SIGN

Smithard D, O'Neill P, Park C, Morris J, Wyatt R, England R, Martin D (1996) Complications and outcome after acute stroke. Stroke 27:1200-1204

Sørensen RT, Rasmussen RS, Overgaard K, Lerche A, Johansen AM, Lindhardt T (2013) Dysphagia screening and intensified oral hygiene reduce pneumonia after stroke. J Neurosci Nurs 45:139-146

Wagner C, Marchina S, Deveau JA, Frayne C, Sulmonte K, Kumar S (2016) Risk of strokeassociated pneumonia and oral hygiene. Cerebrovasc Dis 41:35-39

Warnecke T, Im S, Kaiser C, Hamacher C, Oelenberg S, Dziewas R (2017) Aspiration and dysphagia screening in acute stroke-the Gugging swallowing screen revisited. Eur J Neurol 24:594-601

Wilson RD, Howe EC (2012) A cost-effectiveness analysis of screening methods for dysphagia after stroke. PM\&R 4:273-282

Winstein CJ, Stein J, Arena R et al (2016) Guidelines for adult stroke rehabilitation and recovery. Stroke 47:e98-e169

Yang SN, Pyun S-B, Kim HJ, Ahn HS, Rhyu BJ (2015) Effectiveness of non-invasive brain stimulation in dysphagia subsequent to stroke: a systemic review and meta-analysis. Dysphagia 30:383-391

Ye Q, Xie Y, Shi J, Xu Z, Ou A, Xu N (2017) Systematic review on acupuncture for treatment of dysphagia after stroke. Evid Based Complement Alternat Med 2017:6421852

Zhang JH, Wang D, Liu M (2014) Overview of systematic reviews and meta-analyses of acupuncture for stroke. Neuroepidemiology 42:50-58

Zhang M, Tao T, Zhang Z-B et al (2016) Effectiveness of neuromuscular electrical stimulation on patients with dysphagia with medullary infarction. Arch Phys Med Rehabil 97:355-362

Open Access This chapter is licensed under the terms of the Creative Commons AttributionNonCommercial-NoDerivatives 4.0 International License (http://creativecommons.org/ licenses/by-nc-nd/4.0/), which permits any noncommercial use, sharing, distribution and reproduction in any medium or format, as long as you give appropriate credit to the original author(s) and the source, provide a link to the Creative Commons license and indicate if you modified the licensed material. You do not have permission under this license to share adapted material derived from this chapter or parts of it.

The images or other third party material in this chapter are included in the chapter's Creative Commons license, unless indicated otherwise in a credit line to the material. If material is not included in the chapter's Creative Commons license and your intended use is not permitted by statutory regulation or exceeds the permitted use, you will need to obtain permission directly from the copyright holder. 